\title{
Server hardware resources optimization for virtual desktop infrastructure implementation
}

\author{
K. Makoviy ${ }^{1}$, D. Proskurin ${ }^{1}$, Yu. Khitskova ${ }^{1}$, Ya. Metelkin ${ }^{1}$ \\ ${ }^{1}$ Voronezh State Technical University, 20 let Oktyabrya str., 84, 394006, Voronezh, Russia
}

\begin{abstract}
A new model of capacity planning problem applied to Virtual Desktop Infrastructure implementation is proposed. The possibility of applying the methods of integer mathematical programming to the problem of optimizing server set providing the predetermined number of virtual machines operating.
\end{abstract}

Keywords: capacity planning; virtual desktop infrastructure; integer programming; server hardware; equipment costs

\section{Introduction}

Virtualization is a common concept for concealing the real structure that is used for creating virtual hardware and operating system, virtual storage and network resources. Most organizations of different sizes and income have implemented server virtualization over the past 10 years. Server virtualizations is based on the hypervisor technology, which creates a thing interlayer between hardware and guest operation system.

On the next step of developing IT infrastructure, organizations address to the technology of centralized desktop execution enhancing end user experience and IT management of desktops. While implementing desktop virtualization it is essential to understand that this solution requires not only adequate planning but also significant financial costs. Value of hardware for physical servers makes considerable contribution for the investment costs [1] whereas optimal configuration of the servers purchased can save considerable funds.

We offer a mathematical model for solving the optimization problem of server resources needed for desktop virtualization implementation and present computing results.

\section{The object of the study}

Server virtualization is essentially a server consolidation, i.e. an approach to the efficient usage of physical server resources. This technology allows several operation systems to run on one physical server and isolate applications from each other's influence, minimize investment and operational costs, avoid overprovisioning.

Desktop virtualization or Virtual Desktop Infrastructure (VDI) uses advantages of server virtualization and cloud technologies bringing together the benefits gained from hypervisor-enabled virtualization and modern display network protocols. Desktop operating systems run on a physical server under control of host operating system i.e. 'hypervisor' whilst screen image is delivered by a network protocol to a client device which may be a Personal Computer (PC), Thin Client, laptop, tablet, etc. There are several commercial software products for implementing Virtual Desktop Infrastructure. The most popular ones are VMWare Horizon View, Microsoft VDI, XenDesktop from Citrix. There is also freeware product on the base of Linux KVM.

One of the key perspectives of VDI implementation is a possibility to execute any application on any device for which there is a VDI client since applications are executed on the operating system running on the server, not on the device itself. Thus, desktop virtualization provides the basis for extremely promising technology allowing creation a common learning environment - BYOD (Bring Your Own Device) - a new initiative giving opportunity to use wide variety of client's personal devices in a corporate environment.

The number of client computers in a typical organization far exceeds the number of servers therefore this is so important to be able to assess server resources required to run client virtual machines. No less important is to be able to choose the optimal set of hardware servers, for example, from the range of particular vendor. The key moment to minimize expenses of hardware procurement is a clear view of hardware server set needed to provide execution of required number of virtual desktops. We consider VDI implementation in a high school institute, namely the Voronezh State Technical University, which has already a centralized server infrastructure and well-designed network. Desktop infrastructure in an educational institution contains as a rule several sets of identical computers that placed in computer labs. Definitely apart from desktops in computer labs there are a large number of computers with diverse software, which are used by staff. This type of computers is not the best choice to being virtualized at the first stage of the project. In our model, we assume a number of identical desktops that should be placed optimally on hardware servers. Identity we understand like equivalence in their performance requirements specifically memory needs for running desktop applications. 


\section{Methods}

The problem of virtualized server optimization was considered previously in two aspects - static and dynamic. Static Server Allocation Problem is an approach based on a service concept, the model was introduced in [2] and designed to optimally allocate source servers to physically target servers and was proven that this model is NP-hard problem, heuristic solution based on bin packet problem is offered. Another option of using linear programming methods for virtualized system placement representing the dynamic aspect of the problem is used for creating application placement controller pMapper [3].

There are several attempts to solve the problem of dynamic replacement of virtual machines on existed physical server infrastructure in datacenter to optimize energy consumption, minimize administrative efforts, increasing server utilization. An approach of dynamic resource allocation for large Internet-oriented data centers bases on queuing theory and Erlang's loss formula represented in [4]. On the other hand it is proposed to use a genetic algorithm based approach, namely GABA, to adaptively self-reconfigure the VMs (Virtual Machines) in large-scale data centers [5]. All the models proposed focuses on the server virtualization not the desktop virtualization. As for desktop virtualization an allocation algorithm based on a bin-packet problem is developed [6]. It is mainly focused on achieving a balance between resource usage optimization and user satisfaction.

In this work we concentrated on the problem of server hardware assessment optimization in order to reduce financial costs while implementing desktop virtualization at the university. To achieve this goal we have to analyze resource requirements of VMs that will be used, number of VMs, and range of hardware servers of the vendor then solve optimization problem to choose a set of optimal server models and their configuration to minimize total cost.

\subsection{Model description}

For the model we assume a particular number of the same virtual desktops. We plan to use them for computer labs at the university and actually we probably will have a need of several types of virtual machines for different labs but for the first approximation, we will consider all virtual machines have exactly the same resources requirements.

We consider discrete set of server platform models, each of them may be supplemented by additional RAM (Random Access Memory) modules. We can extend RAM with additional memory modules that have various amounts and prices. We assume also that performance of the server is acceptable if RAM amount is sufficient for running VMs only in virtual memory not using as a rule a paging file. In this approximation, we do not consider the processor load since the main purpose of this model is minimizing total costs at the very start of VDI implementation project.

For the model description, we introduce the following variables:

$\bar{S}=\left\{S_{1}, S_{2} \ldots S_{m}\right\}$ - vector of server platform models that can be used for the hardware servers, where $m$ - total number of server platform models selected for consideration;

$\bar{C}=\left\{C_{1}, C_{2} \ldots C_{m}\right\}-$ vector of values of server platforms $\bar{S}$, where $C_{i}$ - is a value of $S_{i}, S_{i} \in \bar{S}, i=1 . . m ;$

$\bar{N}=\left\{N_{1}, N_{2} \ldots N_{m}\right\}$ - numbers of servers of server platform model $S_{i}$ that will be used in a final set;

$\bar{P}=\left\{P_{1}, P_{2} \ldots P_{m}\right\}$ - vector of memory slots in the server $S_{i}$, this is a maximum number of memory modules that can be used for the server $S_{i}$;

$\bar{M}=\left\{M_{1}, M_{2} \ldots M_{m}\right\}-$ vector of maximum RAM amounts that can be added to the server platform $S_{i} ;$

$\bar{R}=\left\{R_{1}, R_{2} \ldots R_{k}\right\}$ - amount of memory module $j, j=1 \ldots k$, where $\mathrm{k}-$ is the number of types of RAM modules;

$\overline{C v}=\left\{C v_{1}, C v_{2} \ldots C v_{\mathrm{k}}\right\}$ - value of memory module $j, j=1 . . k$.

Because our goal is to minimize costs then we determine an objective function reflecting the total cost of the hardware server set. The total cost of the server consists of the value of based server platform model $\left(C_{i}\right)$ and the cost of additional RAM modules $\left(\sum_{j=1}^{k} C v_{j} n_{j i}\right)$, where $n_{j i}$ - number of RAM modules $j$ on the server $S_{i}$. Thus, the objective function is the following:

$$
F=\sum_{i=1}^{n}\left(C_{i}+\sum_{j=1}^{k} C v_{j} n_{j i}\right) N_{i}
$$

In the following we present constrains for the objective function:

1. The total amount of RAM should not exceed the one supported by this server platform model:

$$
\sum_{\mathrm{j}=1}^{k} R_{j} n_{j i} \leq M_{i}
$$


where $n_{j i}$ - number of RAM modules $j$ on the server $S_{i}, j=1 . . k, i=1 . . m$.

2. The total number of RAM modules cannot exceed the number of hardware server model memory slots:

$$
\sum_{j=1}^{k} n_{j i} \leq P_{i}
$$

3. The total amount of RAM memory on all servers out of server set should provide enough memory to run necessary number of VMs:

$$
\sum_{i=1}^{n}\left(\left[\sum_{j=1}^{k} R_{j} n_{j i}\right] / V\right) \geq N_{V},
$$

where $N_{V}$ - is a number of VMs, $V$ - memory needed for one virtual machine.

4. To get a solution that makes a sense we will add a constrains for numbers of servers and RAM modules to be integer:

$$
N_{i}, n_{j i} \geq 0, \mathrm{i}=1 . . m, j=1 . . k, N_{i}, n_{j i} \text {-integer }
$$

The model proposed makes it possible to solve the problem of selecting the optimal set of server hardware equipment necessary for Virtual Desktop Infrastructure deployment. This model can be refined to allow sets of virtual machines that differs by hardware resources requirements and expand the range of considered hardware resources types.

\subsection{Model solution.}

In order to obtain a solution we divided this problem into two parts:

1. On the first step of calculation, we create optimal filling of the server slots by RAM modules, analyzing filling for $25 \%$, $50 \%, 75 \%$ and $100 \%$ of the maximum amount. Objective function $C_{v_{i}}^{p}$ reflects the cost of RAM added to the $S_{i}$ server platform model filled with RAM modules by part equal to $p$ of the maximum and is the following:

$$
C_{v_{i}}^{p}=\min \sum_{j=1}^{k} C v_{j} n_{j i}
$$

subject to:

$$
\left\{\begin{array}{c}
\sum_{j=1}^{k} R_{j} n_{j i}=M_{i} p \\
\sum_{j=1}^{k} n_{j i} \leq P_{i}
\end{array}\right.
$$

where $p$ - part of the maximum RAM amount, which can be either $0,25,0,5,0,75$ and 1 . This is linear programming problem, which was solved by branch and boundary method [7]. For each filling percentage, we get the optimal set of memory modules for every server platform model. Thus, we get four hardware servers for selection instead of one server platform model.

2. On the second step we form a final set of servers minimizing the following objective function:

$$
\min \sum_{i=1}^{m} \sum_{j=1}^{4}\left(C_{i}+C_{v_{i}}^{p_{j}}\right) N_{i j},
$$

where $C_{v_{i}}^{p_{j}}$ is a result of (6), i.e. cost of additional memory of server $S_{i}$, filled by memory modules on $p_{j}$ part, subject to (4) and $N_{i j} \geq 0, i=1 . . m, j=1 . .4, N_{i j}$-integer .

\section{Results and Discussion}

In the following, we provide the numerical results of applying this model to a set of servers for deploying different numbers of virtual machines. Any set of hardware server platforms by one vendor can be used as an initial set of server platform models. The servers used in calculation presented in the table 1. These are 11 models of HP ProLiant Servers of ML product line. One of the main reasons to use these servers was the fact that HP ProLiant Servers are used in Voronezh State Technical University ITinfrastructure. The cost and configuration of server platform models were taken from the site of one of the server distributors [8]. It happens that two models differ only in the processor that is why CPU model is also presented in a table 2.

For the problem solution, we used MatLab realization of the brunch and bound method [7]. As for amount of memory needed for one virtual machine we assume it is $4 \mathrm{~Gb}$. This the amount recommended by vendors of VDI software is used as a first approach. Further investigations of the memory amount necessary for one virtual machine should base on performance counters analysis in a pilot project. Some software products can help to estimate the required amount of memory, for example, VMWare View Planner.

There are five types of RAM modules available for HP ProLiant Servers: $2 \mathrm{~Gb}, 4 \mathrm{~Gb}, 8 \mathrm{~Gb}, 16 \mathrm{~Gb}, 32 \mathrm{~Gb}$ value $26,136,215$, 315,840 USD respectively. For each server platform model the problem of optimal memory filling up to $25,50,75$ and $100 \%$ of 
Mathematical Modeling / K. Makoviy, D. Proskurin, Yu. Khitskova, Ya. Metelkin

maximum amount is resolved. It was not always possible to get $100 \%$ of maximum possible memory capacity because of the pre-installed small RAM modules. In this case, the maximum possible amount of memory was considered. The result of optimal filling the server slots by RAM modules to minimize cost while maximizing the amount of memory is in Table 2 . Table 1. Initial set of hardware server models.

\begin{tabular}{|c|c|c|c|c|c|c|c|}
\hline & Name & $\begin{array}{l}\text { Initial } \\
\text { RAM } \\
(G b)\end{array}$ & $\begin{array}{l}\text { Number } \\
\text { of RAM } \\
\text { modules } \\
\text { (pcs.) }\end{array}$ & $\begin{array}{l}\text { Max amount } \\
\text { of RAM }(\mathrm{Gb})\end{array}$ & $\begin{array}{l}\text { RAM slots } \\
\text { (pcs.) }\end{array}$ & CPU & $\begin{array}{l}\text { Cost } \\
\text { (USD) }\end{array}$ \\
\hline
\end{tabular}

\begin{tabular}{|c|c|c|c|c|c|c|c|}
\hline 1 & ML150 Gen9 NHP & 4 & 1 & 512 & 16 & E5-2603v3 - 1.60 & 1580 \\
\hline 2 & ML150 Gen9 Hot Plug & 8 & 1 & 512 & 16 & E5-2609v3 - 1.90 & 1700 \\
\hline 3 & ML150 Gen9 NHP & 8 & 1 & 512 & 16 & E5-2609v3 - 1.90 & 1960 \\
\hline 4 & ML350p Gen8 & 8 & 2 & 384 & 24 & E5-2620 - 2.00 & 3300 \\
\hline 5 & ML350p Gen8 & 8 & 2 & 384 & 24 & $\mathrm{E} 5-2630-2.30$ & 4300 \\
\hline 6 & ML350p Gen8 & 32 & 4 & 384 & 24 & E5-2620-2.00 & 4440 \\
\hline 7 & ML350e Gen8 Hot plug & 8 & 2 & 192 & 12 & E5-2420-1.90 & 1874 \\
\hline 8 & ML350p Gen8 E5-2620 Hot Plug & 16 & 2 & 384 & 24 & E5-2620-2.00 & 3556 \\
\hline 9 & ML350p Gen8 E5-2620 & 8 & 2 & 384 & 24 & E5-2620 - 2.00 & 3169 \\
\hline 10 & ML350e Gen8 Hot plug & 2 & 1 & 96 & 12 & E5-2407-2.20 & 1624 \\
\hline 11 & ML350p Gen8 HPM & 16 & 2 & 384 & 24 & E5-2640v2 - 2.00 & 7100 \\
\hline
\end{tabular}

Table 2. Optimal filling of server slots for several server platform models.

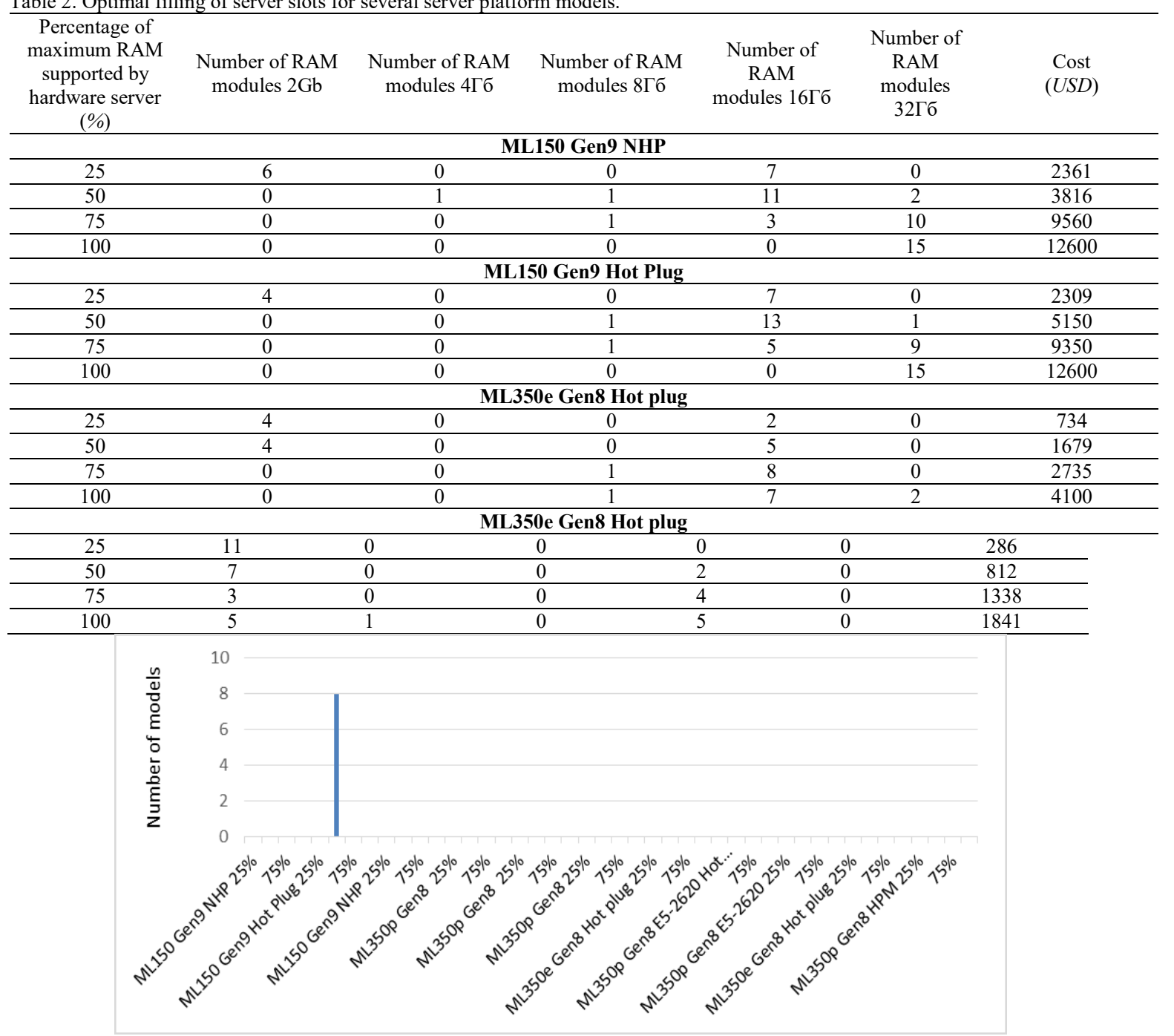

Fig.1. Problem resolution for 500 virtual machines. 
The obtained results are used in the second part of solution that implement selection of optimal final set of servers from the variety of servers determined on the first part of solution. The result of model solution for 500, 700, 900 and 1000 VMs is presented on the figures 1-4.

According to figure 1 for placing 500 virtual machines, it's optimal to use eight servers ML150 G9 Hot Plug with 50\% of memory filling. Table 2 shows that for this filling it is necessary to add to the base model one RAM module of amount of $8 \mathrm{~Gb}$, 13 modules of $16 \mathrm{~Gb}$ and one of $32 \mathrm{~Gb}$.

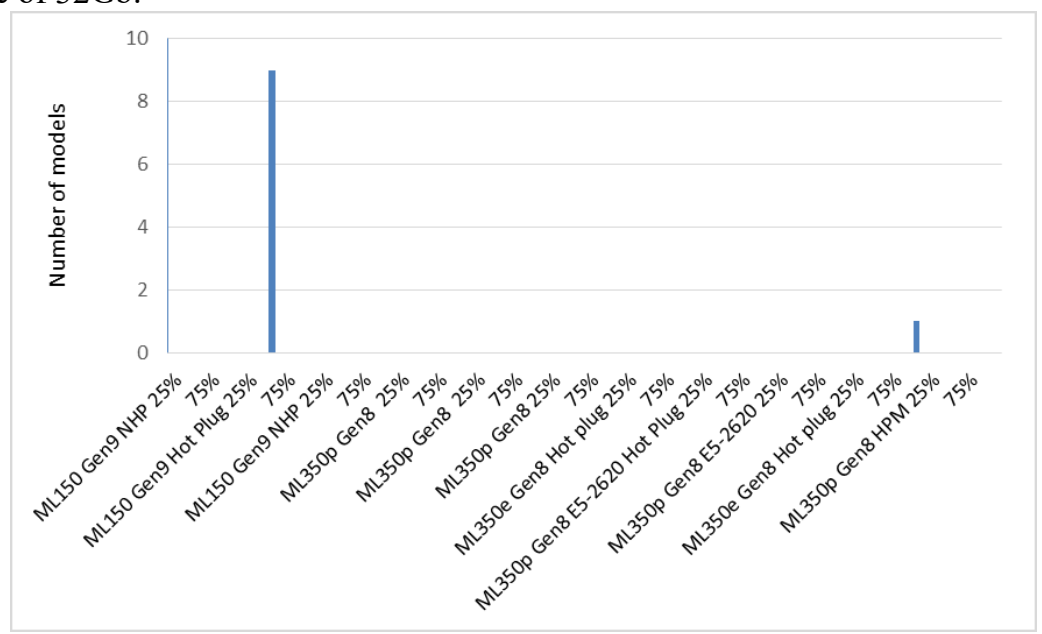

Fig.2. Problem resolution for 600 virtual machines.

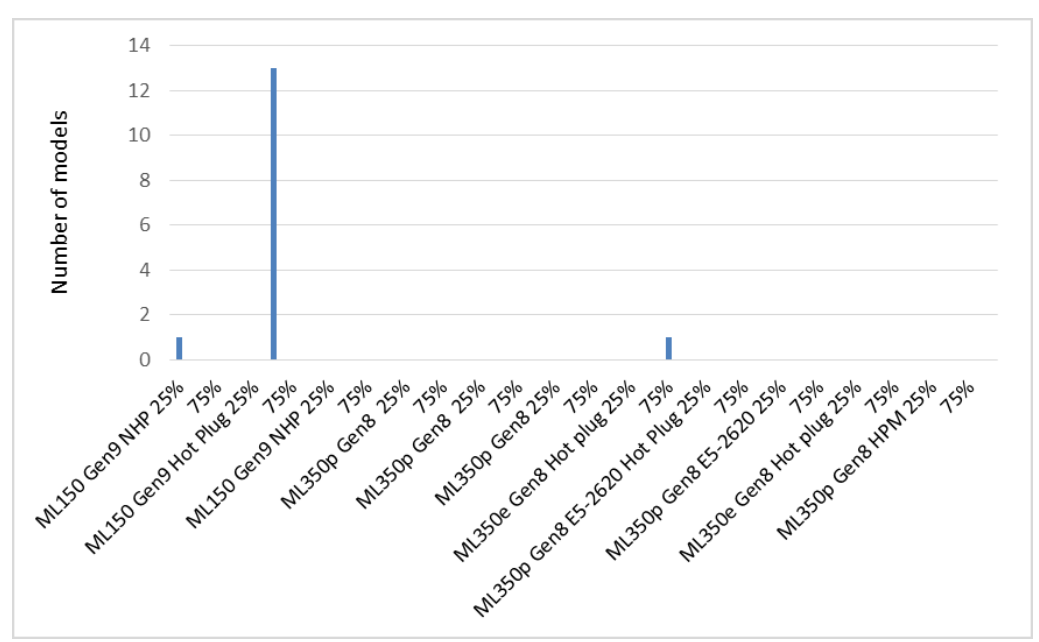

Fig. 3. Problem resolution for 900 virtual machines.

For 600 virtual machines it's optimal to use nine servers ML150 G9 Hot Plug with the following set of RAM modules: $1 \mathrm{x}$ $8 \mathrm{~Gb}, 13$ x 16Gb and 1 x 32Gb and one server ML350e G8 Hot Plug with RAM: 5 x 2Gb, 1 x 4Gb and 5 x $16 \mathrm{~Gb}$.

For 900 virtual machines the following final is obtained (figure 3, table 2):

- 1 x ML150 G9 NHP server model with RAM modules:

- $6 \times 2 \mathrm{~Gb}, 7 \times 16 \mathrm{~Gb}$;

- 13 x ML150 G9 Hot Plug server with RAM:

- $1 \times 8 \mathrm{~Gb}, 13 \times 16 \mathrm{~Gb}, 1 \times 32 \mathrm{~Gb}$;

- 1 x ML350e G8 Hot Plug server model with RAM modules listed below:

- $1 \times 8 \mathrm{~Gb}, 8 \times 16 \mathrm{~Gb}$.

For 1000 virtual machines we receive the following result from calculation results presented on figure 4 and table 2 :

- 1 x ML150 G9 NHP server with RAM:

$$
\text { - } 6 \times 2 \mathrm{~Gb}, 7 \times 16 \mathrm{~Gb}
$$

- 14 x ML150 G9 Hot Plug server with RAM:

$$
\text { - } 1 \text { × } 8 \mathrm{~Gb}, 13 \times 16 \mathrm{~Gb}, 1 \times 32 \mathrm{~Gb}
$$

- 1 x ML350p G8 Hot Plug server

- $\quad 4$ × $2 \mathrm{~Gb}, 17 \times 16 \mathrm{~Gb}$.

Analyzing the results received we noticed that server platform ML150 G9 Hot Plug occurs more frequently than the others. This can be interpreted as the server platform model with optimal price quality ratio. This means that an analysis of large number of server platform models can reveal the ones preferred for procurement of equipment in the enterprise.

Application of this model to hardware server procurement can minimize costs during implementation of Virtual Desktop Infrastructure. This model may be extended by considering several types of VMs requiring different amount of memory, taking into consideration processor power and Fault Tolerance demands. 


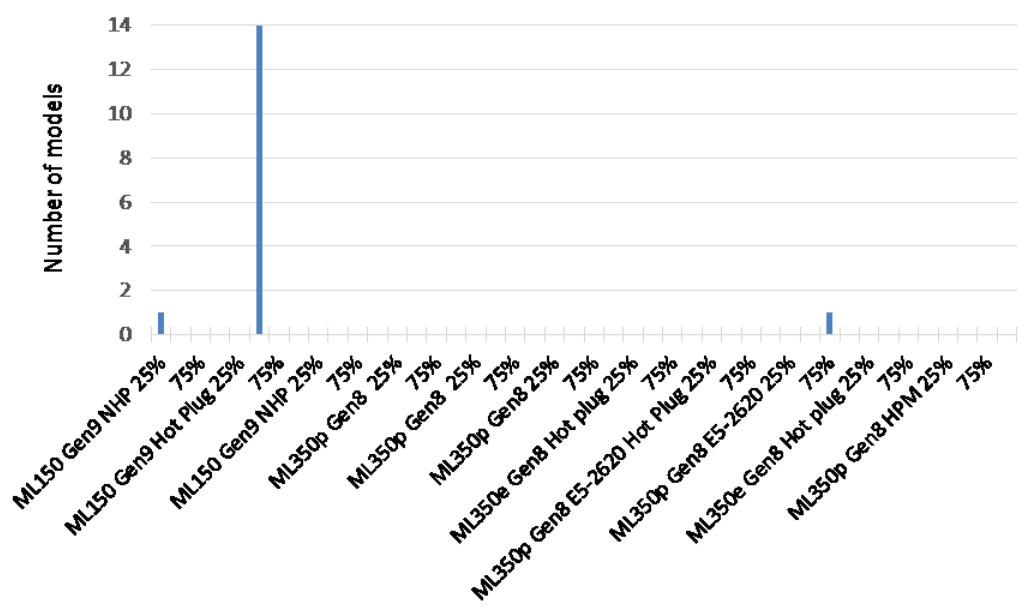

Fig. 3. Problem resolution for 900 virtual machines.

\section{Conclusion}

The interest to VDI technology grows fast because of popularity of cloud computing. Desktop virtualization implementation is a next step in centralizing IT infrastructure that brings both management advantages and academic benefits creating a convenient integrated educational environment. The new model for the optimizing acquisition costs of server hardware purchased for VDI implementation is offered. The results for numerical calculation shows also server platforms models with best price-quality ratio.

\section{References}

[1] Makoviy KA, Khitskova YuV. Econiomic basis of VDI deployment in institution of higher education IT-infrastructure. Modern economy: problem and solutions 2015; 2(62): 75-81.

[2] Speitkamp B, Bichler M. A mathematical programming approach for server consolidation problems in virtualized data centers. IEEE Trans. Services Comput. 2010; 3(X): 266-278.

[3] Verma A, Ahuja P, Neogi A. Mapper: power and migration cost aware application placement in virtualized systems. Proceedings of the 9th ACM/IFIP/USENIX International Conference on Middleware 2008; 243-264.

[4] Song Y, Zhang Y, Sun Y, Shi W. Utility Analysis for Internet-Oriented Server Consolidation in VM-Based Data Centers. Proceedings of the IEEE International Conference on Services Computing 2009; 1-10.

[5] Mi H, Wang H, Yin G, Zhou Y, Shi D, Yuan L. Online self-reconfiguration with performance guarantee for energy-efficient large-scale cloud computing data centers. Proceedings of the IEEE International Conference on Services Computing 2010; 514-521.

[6] Armstrong D, Espling D, Tordsson J, Djemame K, Elmbroth E. Conteztualization: dynamic configuration of virtual machines. Journal of Cloud Computing: Advances, Systems and Applications 2015; 4-17.

[7] Taha HA. Operation research: An introduction. Moscow: Publishing House Willams, 2001; $912 \mathrm{p}$

[8] Servers and Accessories of Hewlett-Packard. URL: http://www.proliant.ru/ files/File/HP_proliant_price_09_15.xls. 